\title{
Modulatory integration: A concept capable of explaining cognitive learning and purposive behavior in physiological terms
}

\author{
RICHARD HIRSH \\ McGill University, Montreal, Quebec, Canada
}

(Invited article)

\begin{abstract}
An argument that the concepts of modulatory synapses and modulatory integration can be used to physiologically explain cognitive learning and purposive behavior is advanced. Modulatory integration is described in formal terms. Cognitive learning is defined as the acquisition of propositional memories or knowledge as opposed to evocative associations. The problem of how propositional learning or knowledge is behaviorally expressed is then considered. It is advocated that propositional learning be regarded as dimensional relations between stimuli. The concept of dimensional relations is closely allied with that of the concept of the transformation of objects, and a dimensional relation can be regarded as implying a transformation. The concept of purposive behavior is used to explain how a particular dimensional relation, implying a particular transformation, gains control over behavior. The formal properties of purposive selection and of modulatory integration are shown to be the same. The process by which an abstract transformation can influence concrete activity is also shown to have the same formal properties as modulatory integration. The concept of modulatory integration can thus be used to explain purposive selection and the influence of transformations upon concrete activity in physiological terms. In an appendix, processes having formal properties like those of modulatory integration are also shown to be useful in constructing dimensions and transformations in the course of experience. Furthermore, dimensions are shown to have the requisite properties for constructing more abstract concepts from less abstract ones. A method in which the construction of dimensions is an intermediate step in constructing more abstract concepts from less abstract ones is presented.
\end{abstract}

Presumably all of the computation that occurs during thought and behavior is done by the nervous system. In this paper, it will be argued that the concept of synaptic modulation, developed by some investigators of the action of peptides within the nervous system, has many of the formal properties of the computation necessary for cognitive learning and purposive behavior, properties that are missing from the classical concept of summational integration. Given the concept of synaptic modulation, it is possible to explain much of cognitive learning and purposive behavior in terms that are quite in accord with current physiological thinking.

Doubtless, many of the psychological processes discussed here are done by large collections of neurons and synapses arranged in particular ways. The point of this paper is that the advent of the concept of synaptic modulation introduces into physiology the kind of computation that is involved in cognitive learning and purposive behavior.

The formal properties inherent in the concept of summational integration are the same as those postulated for the associative processes responsible for the performance

Correspondence may be addressed to Richard Hirsh, Department of Psychiatry, McGill University, 1033 West Pine, Montreal, Quebec H3A 1A1, Canada.

-Accepted by previous editor, Lynn Nadel of already formed associations. Thus, the operation of already formed associations can be physiologically explained in terms of summational integration. According to associative theories, stimuli come to excite or inhibit responses as a result of learning. When the excitatory and inhibitory influences substantially exceed the sum of the influences inhibiting the response, that response will reliably occur. Similarly, the classical concept of integration postulates that the influence of excitatory and inhibitory synapses upon a neuron are summed. When the sum of excitation substantially exceeds the sum of inhibition, the neuron will fire.

The similarity of the formal properties of associative processes and the formal properties of the classical concept of summational synaptic integration is not a coincidence. The classical concept of synaptic integration was formulated to explain certain properties of reflexes by Sherrington, who regarded the reflex as the basic unit of behavior. Associationists regard the association as the basic unit of learned behavior. Pavlov and the early associationists regarded established associations as having all of the properties of reflexes.

In the next two sections, the formal properties of summational integration and an integration in which one input modulates the excitatory or inhibitory effect of another are compared. Then cognitive learning will be defined and 
intuitively related to the concept of modulatory integration and the expression of already acquired cognitive learning. The Appendix deals with the relation between modulatory integration and the acquisition of cognitive constructs.

\section{Summational Integration}

In this section, the formal properties of summational integration will be considered. Eccles's theory of synaptic integration is used as an example, because it is the simplest illustration of the formal properties of summational integration, even though its emphasis on summation at the somatic membrane is clearly not in accord with cabletheory interpretations of such findings as heterogeneous distribution of synaptic terminals, ion channels, and resistances such as dendritic spines.

A schematic representation of summational integration is presented in Figure 1A. Summational integration has four formal properties: (1) With the possible exception of differences in sign (positive or negative), all of the inputs to a summational integration are qualitatively the same. For example, according to Eccles's theory, excitatory synaptic inputs generate intra- and extracellular currents that flow in directions resulting in depolarization of the somatic membrane, and inhibitory synaptic inputs result in currents that are similar in every way except that they flow in directions that result in hyperpolarization of the somatic membrane. (2) The inputs to a summational integration are independent of each other. A particular input makes the same contribution to the output of the integration-the sum-regardless of the other inputs that are present. For example, according to Eccles's theory, a particular synaptic input results in the same current flow whenever it is present, regardless of whatever current

A.

B.

Summational Integration Modulated Integration

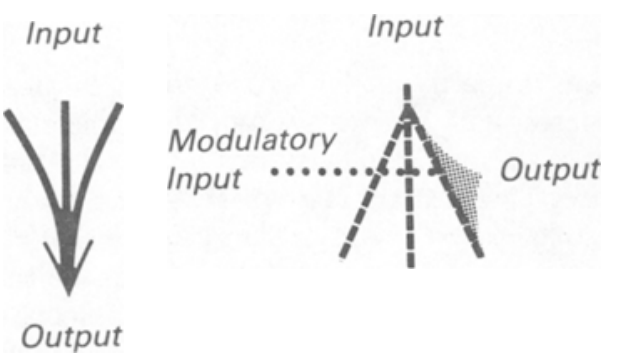

Figure 1. (A) Schematic representation of a summational integration. A number of inputs of the same kind merge to form an output of that kind. (B) Schematic representation of a modulatory integration. A modulated input can give rise to a number of possible outputs of the same kind, as represented by the dashed lines. A modulating input, represented by the dotted line, which is qualitatively different from the modulated input, determines which of the possibilities will actually occur, as represented by the continuous line, and which will not. flows have been initiated by other inputs. (3) The inputs to a summational integration merge together to form the output of the integration. For example, according to Eccles's theory, the flows initiated by the individual synaptic inputs merge to form a net current flow. (4) The output of a summational integration-the sum-is qualitatively similar to all of the inputs. For example, according to Eccles's theory, the net current will either hyperpolarize or depolarize the somatic membrane, just as the currents initiated by the individual synaptic inputs can.

\section{Modulatory Integration}

In recent years, neuropeptides have garnered considerable interest. The term neuromodulation has been coined to denote their actions within the nervous system. The actions of neuropeptides can differ from those of classical neurotransmitters in a variety of ways. As a result, the concept behind the terms remains somewhat ambiguous. Nonetheless, many investigators have concluded that neuromodulation supplements, although by no means supplants, the classical concept of summational integration. The present ideas are based on one of the earliest definitions of a modulator-a synaptic input that has neither direct excitatory nor inhibitory effects but instead alters the effect of another input (Barker \& Smith, 1979). This formulation implies actions by modulators that are formally different from those of classical neurotransmitters. The formal properties of modulatory processes, as defined here, are the same as those of cognitive processes. Should the concept of modulatory processes be valid, it could be used to physiologically explain cognitive processes. The validity of this concept is considered in the last section.

In this paper, a modulator, as just defined, will be referred to as a modulating input. The input that directly results in excitation or inhibition will be referred to as a modulated one, and the interaction between them will be referred to as a modulatory integration.

As just defined, modulatory integrations are formally different from summational ones in a number of ways. First, whereas the inputs to a summational integration are qualitatively similar, the modulated and modulating inputs are qualitatively different. The modulated input has a direct excitatory or inhibitory effect; the modulating input has neither. Second, whereas the inputs to a classical summational integration are independent of each other, the effect of the modulating input upon the resultant output depends upon the modulated input. By examining the output of a modulatory integration, there is no way to determine whether a modulating input is present or not in the absence of an input that can be modulated. If the modulating input alters the effect of the modulated one by $10 \%$, for example, the contribution of the modulating input will vary as the size of the modulated input varies. Third, whereas the inputs to a summational integration merge to form the output, the modulating input alters the effect that the modulated one will have. In this sense, the modulating input does not become part of and remains 
separate from the output of the modulatory integration. Finally, whereas the inputs and the output of a summational synaptic integration are qualitatively similar, the modulating input is qualitatively different from the output of a modulatory synaptic integration. The output of a modulatory integration can be either inhibitory or excitatory, whereas the modulating input is neither.

A modulatory integration is schematically represented in Figure 1B. The modulated input can have a number of different consequences, depending upon the modulating input that is present, as represented by the dashed lines in Figure 1B. In this sense, the modulated input engenders or specifies a set of potential or possible outcomes. The qualitatively different modulating input, represented by the dotted line, determines which of the possible effects of the modulated input, represented by the raised arrow, actually occurs. In a sense, the modulating input refers to or selects the possibility that actually occurs and simultaneously eliminates the other possibilities in the set.

\section{Cognitive Learning:}

\section{Nonassociative and Propositional}

In this section, cognitive learning will be differentiated from associative learning and the concept of modulatory integration will be related to cognitive learning in a quick, intuitive way. Subsequent sections deal with the relation between the two in more detail.

In an association, parts of the nervous system sensitive to the elements of the stimulus are linked to the parts producing a response. The neural elements sensitive to the stimulus, and thus the stimulus itself, are most definitely part of the association. Various segments of the link may be excitatory, evoking a response in the next stage of the chain, or inhibitory, countering the evocation of a response in the next stage. When the total excitation of a particular response substantially exceeds the total inhibition of it, that response will reliably occur. The association, in such a case, is said to be strong. If the stimulus strongly excites competing responses, they will strongly inhibit or interfere with each other, and none of them will reliably occur in the presence of the stimulus. To say that a stimulus is strongly associated with more than one response is a contradiction in terms in the context of associative psychology.

Adding S-S elements, in which parts of the nervous system sensitive to one stimulus come to excite or inhibit parts initially sensitive only to another stimulus, does not change the formal properties of the underlying physiological mechanism in any way. The processes by which a stimulus excites or inhibits activity in a part of the nervous system sensitive to another stimulus are no different from the processes by which it excites or inhibits activity in a part of the nervous system producing an overtly observable response. In both cases, the outcome is entirely determined by the stimulus that is present, and summational integration is sufficient to determine the effect that the diverse elements of the stimulus that are present will have.

Cognitive learning is propositional or representational rather than associative. The organism perceives and remembers the way in which parts of the world are related to each other. The information in memory is knowledge that does not necessarily dispose the animal to act in a particular way; it is pure knowledge.

To illustrate, consider the concept of a cognitive map, which was developed by Tolman (1948), one of the first experimentalists working with animals to advocate a cognitive approach to learning. Tolman thought that learning resulted in cognitive maps analogous to geographic maps. The lines that can be drawn on a geographic map represent relations between points in terms of distance and direction. Similarly, in cognitive learning, the acquired information represents the way parts of the world are related to each other. The activity of neuronal elements mapping the relation could represent it to the rest of the nervous system in the same way that a line detector as postulated by Hubel and Wiesel (1974) could represent a line in a particular orientation to the rest of the nervous system.

If all inputs to and within the nervous system resulted only in synaptic excitation or inhibition, as opposed to some form of modulation, the idea of a propositional or representational memory, as opposed to an associative one, would be physiologically untenable. The only way a memory could be expressed would be if it were excited by a stimulus and it in turn excited or inhibited a response. There would be no point in speaking about it as anything other than associative.

However, the concept of modulation has changed the situation considerably. Memory is often spoken of being in storage, and of being unable to influence behavior until it is retrieved from storage. Note that retrieval implies that the memory has either been transferred to an active place or put in an active state. Suppose retrieval, and thus the capability of influencing behavior, was crucially dependent upon modulation. Now the expression of the memory would not be entirely dependent upon the excitatory and inhibitory inputs it received.

\section{Purposive Selection of Information for Behavioral Expression}

Even when these last points are grasped, a number of psychological questions remain to be answered in order to explain how propositional or representational memories are behaviorally expressed. One such question is how a particular memory is selected from among all of the others that are available in storage to be expressed. Because memories in storage are unable to influence behavior, there is no need to limit the number of memories representing different relations between one stimulus and other parts of the world. The presence of the stimulus would not automatically result in conflict, as would be 
the case if the stimulus was part of more than one association of roughly equal strength. In a cognitive system, the presence of a stimulus is not sufficient to ensure the expression of a memory. Something must retrieve the memory, making it capable of influencing behavior.

The concept of purposive selection can be utilized to explain how appropriate memories are selected from among those available for expression. A purposive selection is one that is at least partially determined by the end that is to be achieved by the resultant behavior. Thus, advocates of purposive selection assume that relations between potential incentives and other parts of the world are perceived and remembered. Note that it is the relation between the incentive and some other part of the world, not the motivational state that it satisfies, that is remembered.

Motivational states, when present, are assumed to place a positive value upon the incentives that satisfy them and also, by extension, upon the memories representing such incentives. In the vernacular, motivational states specify what is wanted. The stimuli, in turn, specify what is possible. Only memories that represent both a stimulus that is present and an incentive that has a positive value at the moment are selected for expression. In a sense, the motivational state, through its action upon the incentive represented within the recovery, retrieves the memory.

One of the first experimental psychologists to advocate purposive selection was Tolman (1932). The associationists of that time also thought that motivational states played a role in selecting learning for performance, but in a way that was formally different. Motivational states were assumed to be no different from external stimuli in the way they participated in the selective process. When present, their excitatory or inhibitory influence was added together with that of the external stimuli present to determine whether the successive stages of the association operated or not. That contemporary associationists have assumed that motivational states affect incentives, and thereby affect the selection of learning for expression in the same way as purposive selection, does not change the formal properties of the process in any way.

The formal properties of purposive selection of representational memories for behavioral expression are inherent in the concept of modulatory integration, as illustrated in Figure 2. A stimulus that is represented in many different relations is analogous to a modulated input in that it can lead to many different possibilities. The many different relations that might be expressed are analogous to the many different possibilities engendered by a modulated input. Just as a modulating input is qualitatively different from a modulated one, so too the motivational state is qualitatively different from the stimulus. Just as the modulating input remains separate from the possible outcomes of the integration, so too the motivational state remains separate from any representations that might be selected for performance. And just as the modulating input simultaneously selects some possibilities and rejects

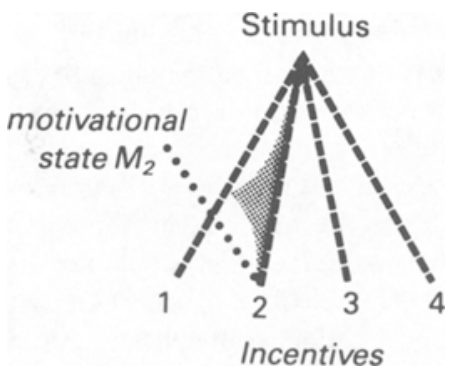

Figure 2. Schematic representation of purposive selection of an acquired relation for behavioral expression as a modulatory process. The stimulus is described in a number of relations, as represented by the dashed lines. Each relation is a candidate for performance. The motivational state, represented by the dotted line, is qualitatively different from the stimulus. By referring to the incentives described within the relations that satisfy it, the motivational state will determine which relations will be expressed, represented by the raised arrow, and which will not.

others, so too the motivational state selects some representations and rejects others. Purposive selection and modulatory integration share the same formal properties. Thus, the formal aspects of purposive selection can be explained in terms of modulatory integration.

Knowing and Doing: Dimensions and Transformations Knowing and doing. Selection of a propositional or relational memory to control behavior is only the first step in the process of how knowing becomes doing. By itself, a relation is incapable of dictating the activity that will change the situation that confronts the animal. Needless to say, animals do carry out activities that change the situations confronting them. Thus, the question of how representations of parts of the world guide such activities arises.

The problem is nicely illustrated by an experiment that was done under Tolman's aegis. Animals learned to negotiate a maze while walking or running through it. The maze was then flooded, so that swimming was the only feasible form of locomotion. If the animals had learned to make running or walking movements at the choice points, that learning would be useless, and additional errors of choice would be expected. However, no more errors of choice were observed than would have occurred if the conditions for locomotion had not changed (MacFarlane, 1930). It was concluded that the animals had learned spatial relations between the start- and endboxes. The question remains as to how such knowledge specifies going from the start- to the endbox. The answer lies in the relation between dimensions and transformation. Dimensions will be discussed first.

Dimensions: In general and in the nervous system. The analogy between geographic maps and cognitive learning may be extended to include dimensions. Geographic maps represent how points in space are related to each other along the dimensions of distance and direc- 
tion. Cognitive learning could represent how stimuli are related along a wide variety of dimensions that would vary depending upon their relevance.

The concept of a dimension is identical to that of a variable as it is used in control theory and robotics. Following the practice used in control theory since its inception, robotics assumes that the situation confronting a robot can be represented by a vector-a set of values along a prespecified set of variables (Albus, 1981). For example, the situation confronting a robot might be described by the vector (dark, rough, and $5 \mathrm{~m}$ north of a particular landmark), values along the variables of illumination, texture, distance, and direction, A dimensional relation between two parts of the world or separate experiences would be the same thing as a difference along one of the variables constituting the vectors that describe each of them. I prefer to use the term dimension rather than variable because it is more graphic and more easily visualized, and thus more readily understood.

Dimensions or variables have a number of key features. They are composed of values, all of which can be applied to the same kind of object. However, the values along a dimension or variable are mutually exclusive. When an object possesses any one of the values along a dimension, it cannot simultaneously possess any of the others. In addition, the values along a dimension or variable are independent of those along at least one other dimension or variable.

Orientation with respect to the horizon is a case in point. A line may have any orientation between $0^{\circ}$ and $180^{\circ}$, but when it has a particular orientation-let us say $45^{\circ}$-it does not have any other. Furthermore, the orientation of the line is independent of its value along some other dimension, such as length.

Most people would hold that dimensions have an additional key feature, the ordering of values along an interval scale. An interval scale is one in which the distance between the values on the scale reflects the real difference between them. Cyberneticists, however, have no problem in speaking about discontinuous variables or dimensions.

In the Appendix, a method for determining whether elements of experience are values along the same dimension is advanced. A very tentative suggestion as to how values might be ordered along an interval scale is also made.

The capacity to utilize dimensions or variables is theoretically within the scope of presently known physiological mechanisms. Characteristics consistent with the key features of dimensions or variables have been observed in various parts of the nervous system. It is well established that some parts of the brain analyze one kind of information, while other parts at the same level of organization analyze different kinds of information. For example, different parts of the prestriate cortex analyze orientation, color, and the direction of movement (Zeki, 1978).

There is also evidence that neurons respond to a particular relation along a dimension or value along a vari- able. Consider the line detectors reported to be present in Area 17 of the striate cortex by Hubel and Wiesel (1962). Individual line detectors respond maximally to straight lines in a particular orientation within their receptive fields. When a straight line in a particular orientation is present, it explicitly excludes the possibility that the line has some other orientation. This exclusion arises from the fact that the receptive field of the detector consists of a linear excitatory core that is flanked by considerably larger inhibitory areas. Thus, if a line with a different orientation were present in the receptive field, the neuron would be inhibited rather than excited.

Other cases of mutual exclusion by values along a dimension should not be hard to find. Generally, the cells within a circumscribed area within a sheet of neurons respond to different stimuli of the same kind. Furthermore, lateral inhibition is particularly prevalent within sheets of neurons. Thus, if the neuron responding to one stimulus property fires, it tends to inhibit its neighbors, which respond to different stimulus properties of the same kind. The tendency to report one value along a dimension is accompanied by a tendency to exclude reports of other values along the dimension or variable. In general, mutual exclusivity can be inferred whenever there is mutual inhibition by neurons responding to different values of the same kind.

Reciprocal inhibition between antagonistic motor neurons can be thought of as constituting a dimension or variable within an effector system. For example, the extension of a joint inhibits flexion of that joint, and vice versa. Thus, extension and flexion of the joint are mutually exclusive and constitute values along a particular variable or dimension to some extent.

Finally, there is also evidence that mutually exclusive values along a dimension or variable can be ordered along an interval scale within the nervous system. Consider the line detectors again. As an electrode is pushed across a column of neurons reporting on the same area of the visual field, the orientation of the line constituting the adequate stimulus changes in an orderly and systematic fashion (Hubel \& Wiesel, 1974). The location of the detectors is ordered and spaced in a way that reflects the differences between the orientations to which they respond.

Transformations. The concept of a transformation is closely allied to that of a dimension or variable. A transformation may be loosely defined as the movement of some object along a dimension or variable or a set of dimensions or variables. More precisely, the state of an object may be thought to be a vector composed of a particular set of properties or values along a particular set of dimensions or variables. When the object is changed or transformed, some of these properties or values will be replaced by other properties or values along the same dimensions or variables. For example, as a balloon is inflated, it goes from having a small surface area to having a large one.

If a transformation is a movement of an object along some dimension or set of dimensions, then a dimensional 
relation or set of dimensional relations can be regarded as a representation of a transformation. Suppose that the knowledge or memory that the startbox of a maze is a certain distance in a certain direction from the location of an incentive has been selected for expression. That memory can serve as a guide as to how the location of the animal is to be transformed, indicating movement in the specified direction for the specified distance. Even though a dimensional relation is purely propositional and is not tightly coupled to a response, it can still guide behavior, albeit rather abstractly, by specifying the effect that the resultant behavior should have. At least some of the gap between knowing and doing has been bridged.

\section{How an Abstract Transformation Influences Concrete Activity}

A third psychological question that arises in trying to understand how representational memories are behaviorally expressed is how the relatively abstract guidance provided by a dimensional relation that has been purposively selected for expression influences concrete activity. To illustrate the problem, consider again the experiment in which the maze was flooded after the animals had learned about it. The results indicate that the acquired information did not specify details of limb movement and muscle contraction, because it guided very different limb movements and muscle contractions equally well. However, a real organism operating in a real world is not going to go anywhere unless it moves its limbs and contracts its muscles in some way. A transformation specifying moving a particular distance in a particular direction makes no suggestion as to how the limbs should move and the muscles should contract.

In this sense, the transformation is qualitatively different than and separate from the concrete activity. Note, however, that in these respects a transformation is analogous to a modulating input, and concrete activity is analogous to the output of a modulatory integration (see Modulatory Integration section above).

Transformations could influence concrete movements in a process that had the same formal properties as modulatory integration, as represented in Figure 3. The mechanical substrate on or in which concrete movements occur can be viewed as an input eliciting a number of possible movements. For example, a watery substrate could elicit a number of possible movements, some locomotory and some not. A solid surface would elicit another set of movements, some locomotory and some not. The transformation could be viewed as a modulating input determining which of the possible movements that the substrate could elicit would actually occur. When a transformation indicating that location should be changed was in control of behavior and the substrate was watery, the movement producing locomotion in water-swimming-would occur. Similarly, if the transformation was operating in the presence of a solid and level substrate, movements producing locomotion-running or walking-would occur. Such a hypothetical process would have the same formal proper-

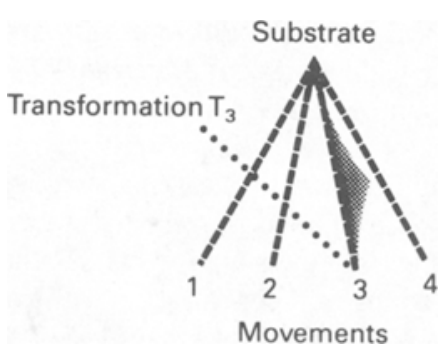

Figure 3. Schematic representation of the process by which a transformation influences concrete activity. A substrate for movement can elicit a number of possible movements, as represented by the dashed lines. The transformation, represented by the dotted line, which is qualitatively different from the substrate for movement, refers to the movement producing it and thus determines which of the movements actually occurs, represented by the raised arrow, and which do not.

ties as modulatory integration, which could be used to physiologically explain the formal aspects of how abstract transformations guide concrete activity.

Once it is understood (1) how a particular dimensional relation can be purposively selected for expression, (2) that a dimensional relation also represents a particular transformation, and (3) how a transformation can influence concrete activity, the question of how representational memory or knowledge is behaviorally expressed is no longer psychologically daunting. Because the formal properties of these hypothetical processes are no different from those of modulatory integration, the question is no longer physiologically daunting, either.

Essentially, I am suggesting that the expression of propositional or representational memories-cognitive learning or knowledge, if you will-can be achieved by a hierarchy of modulatory processes, as illustrated in Figure 4. An input to a given level of the hierarchy would engender a number of different possibilities. A modulating input from a higher level would determine which of these possibilities would actually occur. The output of the higher level would, in turn, act as a modulating input to the next lower level, determining which of the possibilities engendered by an input local to that level would actually occur, and so on.

To state the case in its broadest terms, modulatory integration can be used to provide a physiological explanation of any instance of context dependency. Purposive selection and the influence of a transformation upon concrete activity can be regarded as cases of context dependency. In both cases, one input is ambiguous in the sense that it can result in many different outputs. A contextual input (the motivational state or transformation) is a disambiguator to the extent that it determines which output will actually occur. The idea of context serving as a disambiguator is frequently applied in robotics (see Albus, 1981 , for example). In some cases, an expectation or prediction about the nature of events serves as a context in which actual input is interpreted or to which actual input is compared. Similarly, some learning theorists have 


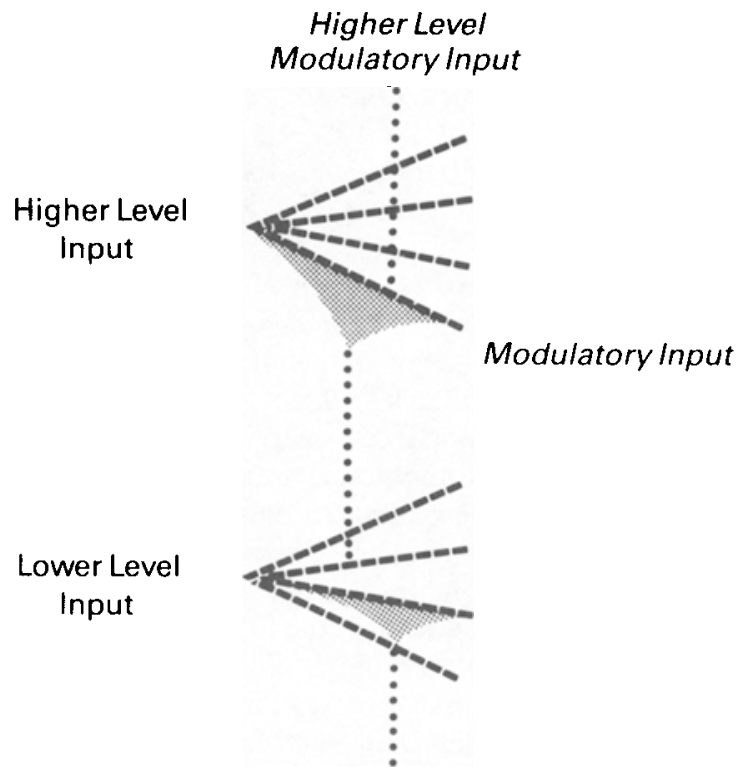

Figure 4. A hierarchy of modulatory processes. A modulating input descends from a higher level to a lower one. It determines which of the possible effects of a modulated input to the lower level will actually occur. The output of the lower level serves as a modulating input to the next lower level, determining which of the possible effects of a modulated input to that level will actually occur, and so on.

begun to speak of context serving to disambiguate stimuli with many possible interpretations (see Bolles, 1985; Nadel \& Willner, 1980; Nadel, Willner, \& Kurz, 1985, for example) and of stimuli that have modulatory effects (see Rescorla, 1985; Jenkins, 1985, for examples).

The influence of context has long been recognized by both associative and cognitive theorists. Associationists regard context as a background stimulus and assume that its influence is addded to that of the more immediate stimulus, much as early associationists assumed that the influence of motivational states was added to that of external stimuli. In some instances in robotics, the influence of the context or background stimuli is added to those of the more immediate stimulus.

However, in cases in which the actual input is interpreted within a context, it is necessary to preserve a distinction between context and the more immediate stimulus or the content. A summational integration cannot embody that distinction. As the old saw has it, one cannot add apples and oranges. However, a modulatory integration involves an interaction between qualitatively different inputs. The modulated input is capable of serving as the content, whereas the modulating input is capable of serving as the context.

\section{The Complementary Effects of Summation and Modulation upon Variation}

In the preceding sections, the utility of summational and modulatory processes in the expression of already acquired learning was discussed. In the following sections, the utility of these processes in the formation of associations will be discussed. The utility of summational and modulatory processes in constructing dimensions and transformations in the course of experience is considered in the Appendix.

Combinations of summational and modulatory processes are useful in forming associations because their effects upon variation are complementary. Summational processes discard the variation between their inputs, which makes them useful in determining central tendencies and common factors. To illustrate, consider the computation of an average of a number of values. The average is considered to be a component common to all of the individual values, which are considered to consist of the average plus or minus some amount of variation. To compute the average, the values are summed. In the course of summation, the variations cancel each other, leaving only the average or common component. The variation has been discarded in the course of summation.

Whereas summational processes discard variation, modulatory ones introduce variation where there is none. When no modulating input channel is present, a given input will always have the same effect or make the same contribution to the output. When a modulating channel is present, the effect of the given input will vary as the modulating input varies.

\section{Modulation and Summation During \\ the Formation of Associations}

Early associative theorists wanted to explain how a stimulus that elicited a variety of responses prior to learning could come to reliably elicit only one of them after learning. To explain the change induced by learning, they tacitly accepted a limited form of modulation. To explain the lack of variation after learning, they relied upon a summational process.

According to associative theories, the tendency of a stimulus to elicit a particular response was deemed to be a function of the strength of the association between them. Reinforcement occurring after the operation of an association resulted in an increase in the strength of the association, whereas nonreinforcement resulted in a decrement.

The roles assigned to reinforcement and nonreinforcement in the learning process are analogous to modulating inputs. By themselves, they have no tendency to excite or inhibit responses. Nonetheless, they alter the ability of associations to excite or inhibit responses. Of late, physiological psychologists have begun to speak of modulatory processes, occurring during storage, that strengthen learning or memories (for examples, see DeWied, 1984; McGaugh, Liang, Bennett, \& Sternberg, 1984; Rosenzweig \& Bennett, 1984), using the term modulation as a substitute for reinforcement.

Note, though, that the modulating effects of reinforcement and nonreinforcement were deemed not to be specific. The strength of any association that had operated just prior to the occurrence of any reinforcer would be increased. Cognitive theories, in contrast, postulate 
specific relations between the motivational states, which exert a modulating influence, and the incentives to which they referred. In retrospect, debate about the existence and nature of reinforcement can be seen as a reflection that summation was the only accepted form of integration. Any form of modulation, no matter how nonspecific, was implausible.

To explain how an association could become so strong that a stimulus consistently elicited only one response, with minimal variation, associative theories postulate a summational process in which the effects of reinforcement and nonreinforcement upon an association accumulate over the course of the animal's life. The strength of the association at any instant was a function of the sum of the effects of all previous reinforcements and nonreinforcements. The sum reflected the central tendency of the entire history of the association. Whether or not the strength of the association had been different in the past was of no consequence to the present or future. Past levels of associative strength were effectively erased.

Theories about purposive behavior are unabashedly modulatory. They usually assume that each motivational state refers to certain incentives and not others, in effect assuming a large number of specific modulators. This view was physiologically untenable when inputs to and within the nervous system were thought to be limited to excitation or inhibition. However, purposive selection did endow the process responsible for assembling performance with sufficient capacity for variation so that it was no longer necessary to postulate reinforcements operating in the aftermath of performance as a special source of variation. In associative systems, behavior is made adaptive in the immediate aftermath of each performance when a response is coupled to stimuli. In contrast, in a cognitive system, behavior is made adaptive just prior to performance. Cognitive theories hold that learning or acquisition of memory is essentially a perceptual process, assuming that any relation that can be perceived can be remembered without any recourse to a special process such as reinforcement.

\section{Current Status of the Concept of Modulators}

Acceptance of the concept of modulatory integration makes physiological explanations of the formal properties of cognitive learning and purposive behavior possible. Modulatory integrations have the formal properties inherent in purposive selection of dimensional relations for behavioral expression and the influence of transformations upon concrete activity.

The formal properties of modulatory integrations are useful in constructing dimensions or variables and transformations from experience, as will be demonstrated in the Appendix. Once present, dimensions can be used both to represent how parts of the world are related to each other and to imply how objects described within those relations are to be transformed.

The question then is, how plausible is the concept of a modulator, which has neither direct excitatory nor in- hibitory synaptic effects, but instead alters the effects of other inputs? The concept was initially advanced because of the large number of molecular species, particularly peptides, that were found to be involved in communication between neurons. It provided roles for all of the players on the stage in a way consistent with the facts of intracellular physiology.

By now, it is clear that not all peptides function as modulators, and the term neuromodulation has acquired other meanings as well. Some peptides induce PSPs that are relatively slow or ultraslow (Libet, 1986). Quite a few molecules involved in interneuronal communication distribute widely and act upon receptors distant from their sources, instead of being released into the synaptic clift. The variety of PSP time courses provides one rationale for a large number of molecules being involved in communication between neurons. Wide distribution provides another. The only way to achieve specificity in a system in which molecular messages are widely distributed is to have a different molecule for each message.

In addition, the conclusion that a molecule has no direct excitatory or inhibitory effects rests upon negative evidence. In many cases, only someone who is thoroughly familiar with the experimental preparation is qualified to judge if direct excitatory and inhibitory effects are truly absent or merely unobserved as a result of faulty technique.

As a result of these developments, some authorities define neuromodulation without referring to the effects of one input upon another. For example, in his text on physiological psychology, Carlson (1986) defines modulators as synaptic inputs acting at some distance from their source. However, Libet (1986), in his review of all aspects of peptidergic action, cites evidence confirming the existence of modulators as defined here. He cites examples of both presynaptic and postsynaptic modulators. Among the postsynaptic modulators are cases of the type discussed here, in which the modulation is present only as long as the inducing molecule is present. He also discusses cases in which the modulation is present long after the inducing molecule has been moved, which could serve as prototypes for reinforcing inputs.

Apparently, the mechanism by which receptors binding benzodiazepines modulate the effect of GABA upon some types of neurons has been established (Haefly, 1984). The only factor that keeps this mechanism from being considered as a case of modulation known to occur in the course of communication between neurons is that the endogenous ligand that is mimicked by benzodiazepines has not been isolated.

Thus, while the concept of a modulator that acts only by altering the effect of other synaptic inputs is by no means universally accepted, it appears to be accepted in many quarters. Physiological psychologists interested in cognitive processes should maintain a keen interest in the resolution of this question. Should the concept of a modulator become accepted doctrine, the formal properties that make cognition so difficult to envision will be inherent 
in one of the elementary integrative processes within the nervous system.

\section{REFERENCES}

ALbus, J. S. (1981). Brains, behavior, and robotics. Peterborough, NH: Byte Books.

BARKER, J. L., \& SMITH, T. G., JR. (1979). Three modes of communication within the nervous system. In Y. E. Erlich, J. Volavka, L. B. Davis, \& E. G. Brungraber (Eds.), Modulators, mediators, and specifiers in brain function: Interactions of neuropeptides, cyclic nucleotides and phosphoproteins in mechanisms underlying neural activity, behavior and neuropsychiatric disorders (pp. 3-26). New York: Plenum.

Bolles, R. C. (1985). A cognitive, non-associative view of inhibition. In R. R. Miller \& N. E. Spear (Eds.), Information processing in animals: Conditioned inhibition (pp. 355-368). Hillsdale, NJ: Erlbaum.

CArLson, N. R. (1986). Physiology of behavior. Boston: Allyn and Bacon.

DEWIED, D. (1984). Neurohypophysical hormones: Influence on learning and memory processes. In G. Lynch, J. L. McGaugh, \& N. M. Weinberger (Eds.). The neurobiology of memory and learning (pp. 289-312). New York: Guilford.

HAEFLY, W. (1984). Actions and interactions of benzodiazepine agonists and antagonists at GABAnergic synapses. In N. G. Bowery (Ed.), Actions and interactions of GABA and benzodiazepines (pp. 263-286). New York: Raven.

Hubel, D. H., \& WIESEL, T. N. (1962). Receptive fields, binocular integration and functional architecture on the cat's visual cortex. Journal of Physiology, 150, 106-154.

Hubel, D. H., \& WiESEL, T. N. (1974). Sequence regularity and geometry of orientation columns in the monkey striate cortex. Journal of Comparative Neurology, 155, 267-294.

Jenkins, H. M. (1985). Conditioned inhibition in key pecking of the pigeon. In R. R. Miller \& N. E. Spear (Eds.), Information processing in animals: Conditioned inhibition (pp. 327-354). Hillsdale, NJ: Erlbaum.

LIBET, B. (1986). Nonclassical synaptic functions of transmitters. Federation Proceedings, 45, 2678-2686.

MacFarlane, D. A. (1930). The role of kinesthetics in maze learning. University of California Publications in Psychology, 4, 277-305.

McGaugh, J. L., Liang, K. C., Bennett, C., \& Sternberg, D. R. (1984). Adrenergic influence on memory storage: Interaction of peripheral and central systems. In G. Lynch, J. L. McGaugh, \& N. M. Weinberger (Eds.), The neurobiology of learning and memory (pp. 313-322). New York: Guilford.

NADEL, L., \&illner, J. (1980). Context and conditioning: A place for space. Physiological Psychology, 8, 218-228.

NADEL, L., Willner, J., \& KURZ, E. M. (1985). Cognitive maps and environmental context. In P. Balsam \& A. Tomie (Eds.), Context and learning (pp. 385-406). Hillsdale, NJ: Erlbaum.

Rescorla, R. A. (1985). Conditioned inhibition and facilitation. In R. R. Miller \& N. E. Spear (Eds.), Information processing in animals: Conditioned inhibition (pp. 299-326). Hillsdale, NJ: Erlbaum.

RosenzweIG, M. R., \& BenNett, E. L. (1984). Basic processes and modulatory influences in the stages of memory formation. In G. Lynch, J. L. McGaugh, \& N. M. Weinberger (Eds.), The neurobiology of learning and memory (pp. 323-332). New York: Guilford.

Tolman, E. C. (1932). Purposive behavior in men and animals. New York: Century.

Tolman, E. C. (1948). Cognitive maps in rats and men. Psychological Review, 33, 189-208.

ZEKI, S. M. (1978). Functional specialization in the visual cortex of Rhesus monkey. Nature, 274, 423-428.

\section{APPENDIX}

In the course of thinking about how already established dimensions and transformations might be expressed in behavior, I had an intuition that modulatory processes could be useful in constructing dimensions and transformations on the basis of experience de novo. Throughout the history of philosophy and psychology, arguments that behavior and thought are cognitive have been equated with arguments that they are innate. I am not particularly competent to review the issue. Nor am I particularly competent to do the formal logic that would constitute a proof that dimensions and transformations could or could not be derived from experience.

However, I do wish to put forth my intuition in the form of a procedure that can be used to establish that sensations and perceptions that have been experienced meet many of the criteria for being values along a dimension or transformation. The procedure will be presented in the context of a particular example for the sake of clarity. In passing, I will add that I do not think that all of the dimensions and transformations used by mature individuals are acquired, but rather that some of them are.

The reader will recall from the section on dimensions that the values along a dimension or variable (1) can all be applied to the same kind of object, (2) are mutually exclusive, and (3) are independent of values along other dimensions or variables. A procedure for determining whether the sensations and perceptions arising during a set of experiences meet these criteria will be presented. In the initial stages of the procedure, a factor common to all of the experiences is isolated in order to determine the kind of object to which the putative values can be applied. Because they discard variation, summational processes are ideally suited to isolating such common factors. In a later phase of the procedure, the "object" common to all of the experiences is made to vary in a number of ways to determine if some values under consideration are mutually exclusive. Because they introduce variation into situations, modulatory processes are particularly useful in this phase.

\section{Formulating Dimensions Based on Experience}

The procedure will be presented by applying it to the experiences denoted in Figure 5A. It is assumed that the system utilizing the method is capable of primitive figure-ground separation, clustering sensations into "objects." In the case of Figure 5A, this assumption implies that four objects will be perceived to be present. Other assumptions required by the procedure will be discussed as needed.

The first major objective in determining whether elements of experience are values along the same dimension is showing that they could all apply to the same kind of object. This can be done as follows. One of the objects present during the experience is selected. In the case of Figure 5A, an object could be selected on the basis of its position in the visual field. Then the properties of the selected object are listed. This procedure is repeated until the properties of each of the objects that is present have been listed separately, as shown in Part 1 of Figure 5B.

Let us say, for example, that round is selected because it is the first item on the first list. This selection, like the ones that preceded it, is analogous to what I have called a modulatory integration. The list is treated as an analogue of a modulated input which could yield three different possible properties, depending upon the list position that was used to select them. List position is treated as an analogue of a modulating input. It is regarded as qualitatively different from both the list and the property that was selected.

The selected property is given a score of 1 every time it is present in one of the lists of object properties. Every list is scanned for the selected property just once. The scores are then summed. This process is repeated for each of the different properties. If the summed score for a property is equal to the 
$A$
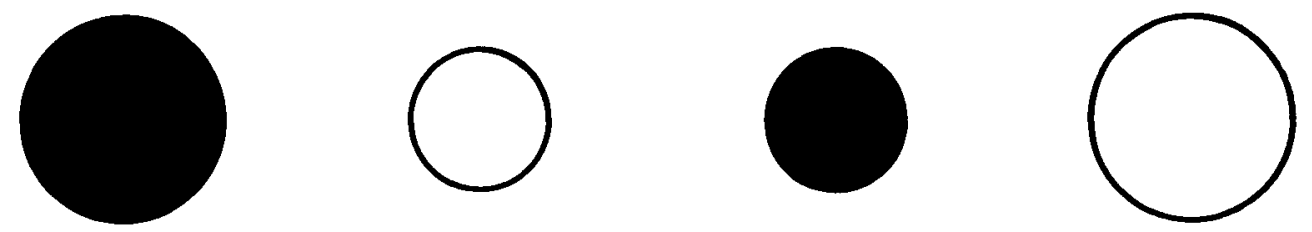

1. properties of the objects

$\begin{array}{llll}\text { round } & \text { round } & \text { round } & \text { round } \\ \text { dark } & \text { light } & \text { dark } & \text { light } \\ \text { large } & \text { small } & \text { small } & \text { large }\end{array}$

2. scores and interpretation

round has a score of 4 . It is common to all of the objects in the sample. If there is a dimension present, it can be a dimension of round objects

small, large, light, and dark have scores between 0 and 4 . They may or may not be values along any dimension that is present.

3. properties of larh, round objects

round round

dark dark

large small

4. scores and interpretation

light has a score of 0. It is excluded by dark and a value

along the same dimension that dark is.

large and small have scores of 1 . They are not excluded by dark and are not values on the same dimension that dark is.

Figure 5. A scheme for constructing a dimension from a set of experiences. (A) A set of experiences from which dimensions will be constructed. (B) The method for constructing a dimension. For explanation, see text.

number of objects sampled, which in the present case is four, the property can be considered to be common to all of the objects in the sample. In the present case, the property round has a score of 4 and is common to all of the objects sampled, as shown in Part 2 of Figure 5B. If there is a dimension or variable present, any of its values could be applied to the kind of object defined by the common property. In the present case, the values of the dimension that is yet to be identified could all be properties of round objects.

A score of less than the number of objects or lists sampled, which in the present case is 1,2 , and 3 , indicates that a property is present in some but not all of the objects being experienced. The noncommon properties are candidates for being mutually exclusive properties in the context of round objects. Determining whether or not some of the noncommon properties are mutually exclusive is the next objective of the procedure.
One of the noncommon properties, for example dark, is selected on the basis of its position in the lists. This noncommon property is used to define a subset of the kind of object that is being considered, in this case dark, round objects. Note that the formulation of the subset is also analogous to a modulatory process. The input round designates four possibilities. A qualitatively different input, dark, is analogous to a modulating input in that it selects some of the possibilities while rejecting the others. As was done to produce the first set of lists, the objects in the subset are selected one at a time and their properties are listed, as shown in Part 3 of Figure 5B.

Next, another property not common to all of the objects in what is now the superset-round objects in the present example-is selected. The members of the subset are now examined for the presence of the newly selected, noncommon property. It is given a score of 1 whenever it is present in a 
member of the subset, and a score of 0 whenever it is absent. Every member of the subset is scanned just once. The scores for each property are summed. Now, however, a score of 0 is considered special. It indicates a property that can be present in the superset-round objects in the present example-but is absent from the subset - dark, round objects. This would be the case for a property that is excluded by the property that was used to define the subset. In the present example, as shown in Part 4 of Figure 5B, the property light is absent from all of the dark, round objects, which would be the case if dark excluded light. This procedure is repeated for each of the noncommon properties of the objects in the superset. In this way, dark would be found to be absent when light was present, large would be found to be absent when small was present, and vice versa. All of the mutual exclusivity present would be identified. In addition, a score greater than $\mathbf{0}$ would indicate a property that was not excluded by the property used to define the subset. In the present example, it would be found that light and dark do not exclude either large or small, or vice versa, as is also shown in Part 4 of Figure 5B. In effect, the values along a light-dark dimension or variable would be found to be independent of those along a large-small dimension or varible, thus fulfilling the third criterion mentioned above.

As outlined to this point, however, the method is quite capable of producing a nonveridical grouping of elements of experience along a dimension or variable. The grouping that the method will produce is, to a large extent, a function of the experiences sampled. Suppose that the objects sampled consisted of two dark, round objects that were large and two light, round objects that were small. In such a sample, there would be a positive correlation between dark and large and also between light and small. Furthermore, large and light would be found to be mutually exclusive, as would small and dark, resulting in a dark/small-light/large dimension, which would be rather counterintuitive. This weakness can be easily corrected by assuming that the system generating the dimension will sample the same kinds of objects many times and that it operates on the principle that if, in any one case, two values are found to be independent, they are always considered to be independent in the context of the kind of objects sampled. Thus, if the sample just hypothesized were examined first, the sample presented in Figure 5A would eventually be experienced also. From then on, large and small would be considered to be independent of dark and light, in the context of round objects.

However, there are other kinds of nonveridical groupings that can emerge from the procedure. One of the properties of intuitively correct dimensions is that the values along them are equally abstract. Light and dark are equally abstract, as are small and large. Unless something is done to ensure that all of the elements of experience being considered as candidates for values along a putative dimension or variable are equally abstract, the method can produce some dimensions that are quite counterintuitive.

For example, suppose the set of experiences sampled consisted of a rock and a pencil. At various levels of abstraction, both of these objects have properties in common. Both are opaque, for example, and any dimension formed might be considered a dimension of opaque objects. Also, at various levels of abstraction, one object has properties that the other does not have, and vice versa. The rock consists of granite, whereas the pencil does not. The pencil has an eraser, whereas the rock does not. As the method stands now, it could produce a granite-eraser dimension for opaque objects, which intuitively is nonsense.
I see no other way to eliminate the inclusion of values from different levels of abstraction along the same dimension except by assuming that (1) one level of abstraction does not begin to develop until the preceding one is more or less complete, rather like floors in a building; and (2) the system constructing a dimension at a given level is constrained to using values from that level.

These assumptions sound ad hoc. However, they reflect what is known about the anatomy and neurophysiology of lemniscal pathways. Usually, at a given level of a lemniscal pathway, all of the neurons are the same number of synapses from the periphery. Because integration occurs only at synapses, all of the neurons at a given level can be expected to be processing equally abstract information. Indeed, the usual finding is that the stimuli adequate for activating the neurons at a given stage of a lemniscal pathway are equal in generality and complexity. Furthermore, a higher level in a lemniscal pathway usually receives all of its input from the immediately preceding level, which ensures that only information from one level of abstraction is being integrated at the next level. In fact, this aspect of the construction of dimensions must be simultaneously addressed with questions about how hierarchies of abstraction develop, which is done below.

A putative dimension or variable would become more general as it was found to apply to more kinds of objects. For example, the light-dark and large-small dimensions could be found to apply to shapes other than round. More importantly, the putative dimension could be applied to relating different kinds of objects to each other. For example, a finding that round objects could be related along a light-dark dimension, combined with one that square objects could be related along the same dimension, would allow round objects to be related to square ones along a light-dark dimension.

The method for grouping values along dimensions was presented here somewhat as if it were a procedure to be executed by a computer. The question of whether it is physiologically plausible in its entirety remains open. The summational processes that were employed are entirely analogous to the classic concept of synaptic integration. The modulatory processes that were employed are analogous to forms of integration being contemplated currently. The other aspects of the procedure, such as listing characteristics of objects, can all be achieved by a human brain that has access to outside resources. Whether they can be achieved by a brain that is limited to internal resources is an open question, but it seems highly likely that they can.

\section{Formulating Transformations \\ Wrought by Concrete Activities}

Most of the steps in establishing the transformation wrought by a given concrete activity that the organism has produced are similar to those in generating a dimension from experience. Thus, the computational capabilities of both conditional and summational operations are useful in determining the transformation wrought by an activity, as they were in determining which elements of experience should be grouped along the same dimension. During the transformation, the state of some object changes, for example, when a lever that is up is pushed down. To establish the transformation wrought by an activity, it is necessary to find the constancy across that activity to determine the nature of the object that has been transformed. For example, a lever is a lever whether it is down or up. Similarly, in establishing a dimension, it is necessary to determine consistency across a set of experiences in order to determine the kind of object to which the dimension can be applied. Moreover, just 
as the values along a dimension are mutually exclusive, so too are the states occurring in the course of a transformation. For example, a lever that is up is not down, and vice versa.

A combination of summational and conditional processes is useful in establishing the transformation wrought by an activity for the same reasons it is useful in generating a dimension. A method for determining the transformation wrought by an activity using a combination of summational and modulatory processes is briefly presented below.

It is posited that the system determining the transformation wrought by a given concrete activity is capable of discriminating between experiences occurring in the course of the activity and remembering the order in which they occurred. It is also posited that a given transformation is constructed on only one level of a system, and that all of the information at that level is equally abstract.

Given these conditions, the determination of the transformation wrought by a given activity begins by using a modulatory process to select one of the experiences occurring during the activity. First, the properties of the selected experience are listed. This process is repeated for each of the experiences. Next, one of the properties is selected, in another modulatory operation, for examination. It is given a score of 1 every time it is present in one of the experiences, and a score of 0 every time it is absent. Each experience is scanned just once. The scores are summed. The properties with scores equal to the number of experiences examined are common to all of them. They can be used to determine the nature of the object that is transformed.

Next, determination of which states are mutually exclusive is undertaken. One of the noncommon properties is selected to serve as a modulating factor in a modulatory process yielding a subset of experiences. Then another property that is sometimes, but not always, present in the superset of experiences is selected in a modulatory process for examining whether it is present in the subset. It is given a score of 1 every time it is present, and a score of 0 every time it is absent. Each member of the subset is scanned for the property just once. The properties with summed scores of 0 are ones that are excluded by the property used to define the subset. When such exclusivity is mutual, some of the states assumed by the object in the course of the transformation have been identified. This process is repeated until all of the mutual exclusivity within the set of experiences has been identified.

The method just outlined is entirely analogous to that for generating a dimension or variable. The employment of modulatory and summational processes is entirely analogous to their employment in the method of grouping elements of experience along the same dimension.

The method rests on the assumption that organisms are inherently capable of ordering experiences in terms of the time of their occurrence. If this assumption is granted, then the order of experience can serve as a basis for ordering values along a dimension. Usually, as objects are transformed, whether due to external forces or the organism's own activity, their states change in a reliable and orderly sequence over the course of time. For example, as an animal eats a supply of food, the size of the pile grows smaller. The states occurring during a transformation are equivalent to the values aiong a dimension or variable. The sequence in which the states occur could be used as the basis for ordering the equivalent values along a dimension.

State changes occurring in the course of a transformation might also be the source of information about spacing values along a dimension. Often, the amount of effort required to transform an object from one state to another along a dimension reflects the difference between the states. When the states are used as values along a dimension, the interval between them might be based on the amount of effort needed to transform an object from one state to the other.

\section{Hierarchies of Abstraction}

In order to avoid inclusion of values from different levels of abstraction along the same dimension, it was necessary to assume that hierarchies of abstraction develop level by level. In this section, I will suggest a method by which dimensions constructed at one level could be used to foster the development of the next level of abstraction, either in the course of an individual's life or in a species in the course of its evolution.

In this method, the construction of a dimension in the context of less abstract categories is an intermediate step in constructing more abstract categories. Then in the context of these more abstract categories, even more abstract dimensions are constructed, and so on. Both modulatory and summational integrations are used in the process.

The basic premise underlying this method is that a dimension can be considered an emergent, higher order property of a category when the members of that category vary along the dimension. I grant that no one individual member of a category possesses a dimension. Each individual possesses only one value along the dimension. Hence, possession of the dimension cannot be considered a criterion for inclusion within a category. Nevertheless, once a category is established on other grounds, the members can be shown to be related to each other along some dimensions. When this happens, the dimensions in question can be considered as properties of the category per se.

The lower line in Figure 6 utilizes the premise advanced in the previous paragraph. Each member of Category A possesses Property A. All of the members of Category A are related to each other along Dimensions $\alpha$ and $\beta$, neither of which includes Property A. Dimensions $\alpha$ and $\beta$ can be considered properties of Category A per se. Similarly, each member of Category B possesses Property B. The members of Category B are related to each other along Dimensions $\alpha$ and $\Delta$, which do not include Property B. These dimensions can be considered properties of Category B per se.

When a dimension is an emergent property of two different categories, it can be considered a property that they have in common. The common emergent property can serve as a criterion for the inclusion of the already established categories in a more abstract category. This premise is utilized on the left side of Figure 6. Category $\alpha$ is, in effect, the set of individuals that can be related to each other along Dimension $\alpha$. This new category is more inclusive than either Category A or Category B. Furthermore, it is defined in a term, $\alpha$, that differs from the terms used to define the antecedent categories, A and B.

Only information about the higher order, relational properties would be carried to the level processing the higher order categories. This premise is illustrated on the left side of the upper line in Figure 6. Only the properties related to each other along the dimensions of $\alpha, \beta$, or $\Delta$ are ascribed to the individual members of a category $\alpha$. Thus, all of the information on the upper level is equally abstract.

By using the procedure outlined in the first section of the Appendix, it can be established that the relational properties $\beta$ and $\Delta$ are mutually exclusive in the context of Category $\alpha$, and can be considered values along a putative dimension $\beta-\Delta$. This is depicted by the middle of the upper line of Figure 6 . The right side of Figure 6 also assumes that the members of another higher order category are also related to each other along the dimen- 


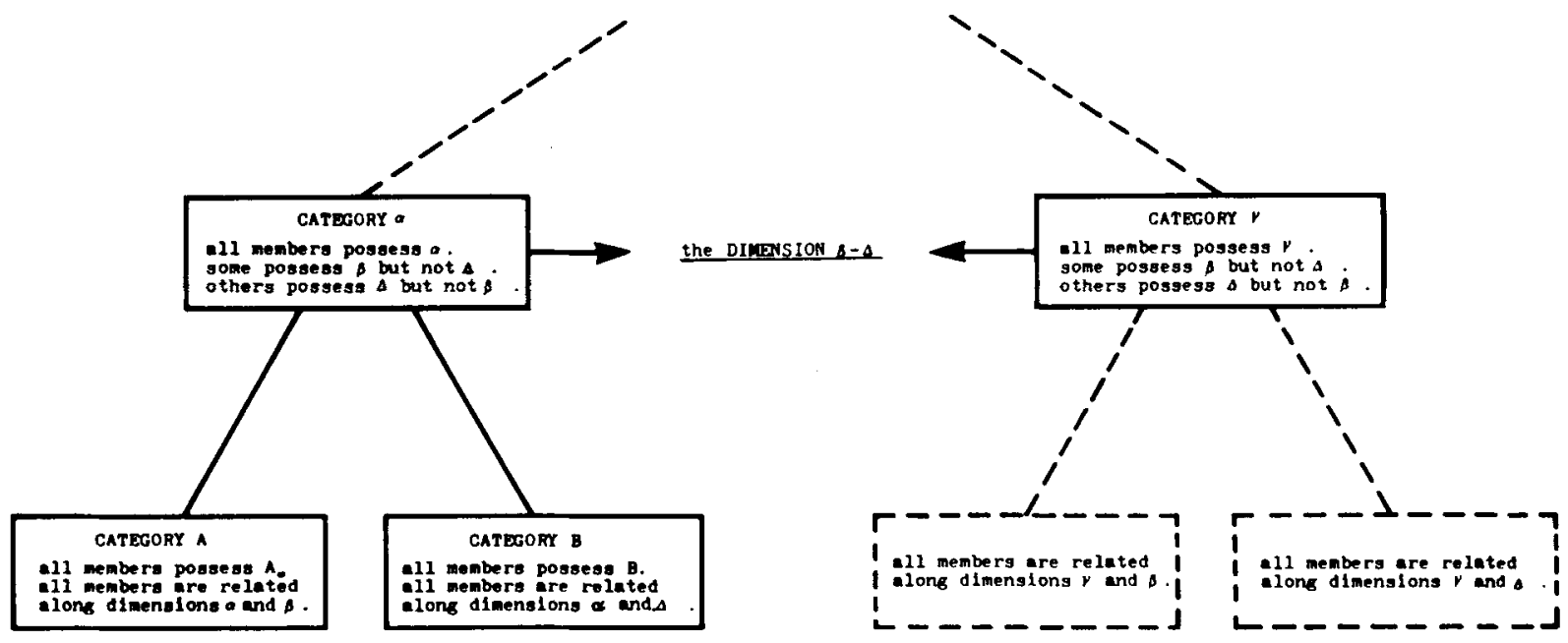

Figure 6. A scheme for constructing more abstract concepts from less abstract ones. Constructing dimensions is an intermediate step in constructing more abstract categories from less abstract ones, and constructing a category is an intermediate step in constructing more abstract dimensions from less abstract ones. For further explanation, see text.

sion $\beta-\Delta$. The common presence of this higher order, dimensional property could serve as the basis for establishing a still more abstract category of individuals related to each other along the $\beta-\Delta$ dimension. This possibility is represented by the dotted lines rising from the upper line in Figure 6.

In this manner, dimensions could be constructed in the context of categories. The resultant dimensions could be considered as emergent properties to be used in constructing or defining even more abstract categories. Then, in the context of the more abstract categories, even more abstract dimensions would be generated, and so on. This could be done in a manner that ensures that all of the information at a given level is equally abstract. The resulting hierarchy would consist of meta level upon meta level.

The problem of abstraction has always been difficult for the associative/empiricist approach. The cognitive/rationalist school has always emphasized that higher order categories are both more inclusive than lower order ones and based on a different order of information. By way of example, consider phylogenetic taxonomy. The phylum Vertebrata includes more species than does the class Mammalia, and the phylum is defined in terms of quite different characteristics.

Associative/empiricist approaches have great difficulty producing abstractions that are both more inclusive and based on a different order of information. One associative approach is generalization. By way of example, generalizing across red, round objects and blue, round objects would yield round objects, a category that is more inclusive than either of its predecessors, but one that is not defined in a new term. Round applies to both of the predecessor categories; the only change was the deletion of red and blue. The second major associative/empiricist approach to abstraction is to hold that lower level concepts are compounded. By way of example, the lower order concepts small, blue, and round would be compounded to form the concept small and blue and round, which is indeed more complex and a different order of information from the antecedents. However, the new concept is not more inclusive than its antecedents. There can be no more objects that are small and blue and round than there are objects that are small, or that are blue, or that are round.

This inability of the associative/empiricists to form higher order categories that are both more inclusive and based on new orders of information is one of the strongest arguments that cognitive/rationalists have for holding that hierarchical abstractions are innate.

Dimensions both introduce a new order of information and are more inclusive than their antecedents. For example, the dimension color includes red, green, blue, and so forth, and refers to the relation between them. I have suggested that dimensions can be constructed from experience, and that this construction can be used as an intermediate stage in constructing hierarchies of abstractions based on experience.

(Manuscript received June 22, 1989;

revision accepted for publication September 11, 1989.) 